\title{
DEVELOPMENT OF LABEL-FREE BIOSENSOR FOR DETECTING STEROID HORMONE CONCENTRATION IN FISH
}

\author{
Haiyun Wu, Hitoshi Ohnuki, TadayoshiMuramatsu, Mutsuko Hirai, Kyoko Hibi, \\ Huifeng Ren, Hideaki Endo*) \\ Graduate School of Marine Science and Technology, Tokyo University of Marine Science and \\ Technology, 4-5-7, Konan, Minato-ku, Tokyo 108-8477, Japan \\ *e-mail endo@kaiyodai.ac.jp
}

\begin{abstract}
A novel label-free immunosensor for detecting steroid hormone was developed. The principle of the sensor system is based on differences in the electrochemical activity induced by an immunoreaction that depends on the levels of steroid hormone in the sample. A gold electrode functionalized with 3-mercaptopropionic acid (MPA) self-assembled monolayer was used to fabricate electrochemical immunosensor. In addition, single-wall carbon nanotube (SWCNT) was selected to expandthe dynamic range of the sensor. The sensor was immersed into a sample solution and measurements were determined by cyclic voltammetry. Each electrochemical measurement including sample immerse only took about $15 \mathrm{~min}$. In this study, we introduced an application of our sensor in detecting 17, 20ß- dihydroxy-4-pregnen-3-one (DHP). The immunosensor showed a specific response to DHP, and the oxidation peak current linearly decreased in the range of 7.8-500.0pg ml-1 (without SWCNT) and 15.6-50000.0pg $\mathrm{ml}^{-1}$ (with SWCNT). The sensor system was then applied to monitor DHP of goldfish (Carassiusauratus) and was compared with the levels of the same samples determined using ELISA as the convention method. Blood plasma of fish was collected every $3 \mathrm{~h}$ after administering a DHP inducer. A good relationship (coefficient: 0.934) was observed between DHP levels determined by both methods.
\end{abstract}

Keyword: Biosensor, Steroid hormone, Immunoassay, Fish, Single-walled carbon nanotube

\section{INTRODUCTION}

In order to meet the needs of health trend and increasing food demands, the consumption of marine products is primarily increasing in the world. But excessive fishing will make the reproduction of marine products becoming harder and eventually lead to a worldwide deficiency of this resource. Like any captive animal, fishes need certain environmental and social cues before they will mature and spawn. Fish farmers need fry or "seed" predictably and in very large numbers. When the numbers of wild-caught fry fall short of what farmers need to stock their ponds or cages, and ultimately to make a profit, producers must somehow induce adult fish to reproduce in captivity. The better the reproductive physiology of fishes is understood, the better are the chances of success with induced reproduction. A complementary approach, where environmental manipulation and more attention to broodstock requirements for diet, water quality, and holding conditions are combined to enhance the outcome of hormone techniques, will be productive.

During the past decade, our knowledge of social and environmental cues in fish reproduction has advanced steadily; at the same time, methods for hormone induced reproduction of fish have become more and more refined. However, in lots of cultured fish, 
systematic production cannot be achieved because the maturation period and ovulation phase are altered by many factors, such as water temperature, light cycle, etc.

Studies about fish reproduction and gametogenesis mechanisms have produced important message.17, 20ß-dihydroxy-4-pregnen-3-one (DHP) was discovered in plasma of the red salmon (Idler et al.1960). It acts as a maturation-inducing hormone in salmonidsand cyprinids. Lou et al. (1984) investigated changes in plasma DHP levels in the rainbow troutOncorhynchusmykissduring the breeding season. In addition, Nagahama and Yamashita (1989) reported that DHP, which is created in follicle cells during the maturation period. In fish, low DHP levels are maintained for several months and then rapidly increase just before ovulation, indicating a relationship between DHP levels and ovulation. Monitoring of DHP levels will be an effective way to predicting ovulation and stabilize seed production of fish.

There are several reported techniques for detecting steroid hormone levels, such as gas chromatography, mass spectrometry, liquid chromatography, and fluorescence-based methods. Though chromatographic and fluorescence-based methods have the advantage of being highly sensitive and specific, but the time-consuming pre-treatment steps and high cost of the instrument make it hard to use for on-site measurement and rapid measurement. Radioimmunoassay and enzyme-linked immunosorbent assay (ELISA)are also used to determine steroid hormone levels. But because of the use of radioactive compounds in radioimmunoassay and high levels of variability, time-consuming complicated steps in ELISA, they are also not wildly used in rapid and safe detection. In this study, we focused on the development of an immunoassay system for steroid hormones. Different from other immunoassay method which needs longer preparation times and multi-reaction steps, label-free immunosensor technique based on highly specific molecular recognition and able to directly detect immune-complexes formed on the modified electrode as a change in electrochemical activity. Therefore, this method is very attractive because of its simple assay procedure that does not require a complicated labeling process.

The development of an electrochemical label-free immunosensor with features such as a single-step process and compact device size would allow for real-time monitoring of steroid hormones at fish farm. In present study, we developed a basic analytical system as the foundation for real-time in vivo measurement of steroid hormone and improved its dynamic range by using single-wall carbon nanotube. The proposed method is based on immunereactions and electrochemical measurements using cyclic voltammetry (CV). For bio-molecular immobilization on the surface of the sensing electrode, we used self-assembled monolayers (SAM) of 3-mercaptopropionic acid (MPA), which are easy to form and stable. In order to increase the dynamic range of sensor and make it possible in no-diluted measurement, we focused attention on carbon nanotubes (CNTs), which are highly conductive. CNTs have excellent chemical and mechanical stability, and their application to the development of highly sensitive immunosensors has been actively studied (Rivas et al. 2007). Specially, single-walled 
carbon nanotube (SWCNT) has various stereo-structures and changeable electronic properties, similar to metals or semiconductor (Saito and Shinohara 2004).

We aimed to develop a novel immunosensor system for steroid hormones. In this study, we introduce a novel way to detect DHPlevels, one of the important steroid hormones in fish plasma. SWCNT was selected to increase the dynamic range of the measurement system. The proposed system is based on immune-reactions and electrochemical measurements using CV. For bio-molecular immobilization on the surface of the sensing electrode, we used SAM. The specificity of the immunosensor was evaluated. The proposed immunosensor system was applied to the measurement of DHP levels in fish plasma samples. The results were compared with those obtained using the conventional ELISA.

\section{MATERIAL AND METHODS}

\section{Reagents}

17 $\alpha$, 20ß-Dihydroxy-4-pregnen-3-one (DHP), 3-Mercaptopropionic acid (MPA), 1-Ethyl3-(3-dimethylaminopropyl) carbodiimide hydrochloride (EDC), N-Hydroxysuccinimide (NHS), Ethylenediaminetetraacetic acid tetrasodium salt hydrate, and Nafion ${ }^{\circledR}$ perfluorinatedionexchange resin were purchased from Sigma (St. Louis, MO). Maturation-inducing steroid (salmonid) EIA antiserum (anti-DHP antibody), maturation-inducing steroid (salmonid) EIA AChE tracer, cortisol EIA standard, estriol EIA standard, testosterone EIA standard, and Ellman's regent were purchased from Cayman Chemical (Ann Arbor, MI). 2-Phenoxyethanol and heparin sodium were purchased from Wako Pure Chemical Industries, Ltd. (Osaka, Japan). SWCNTs were provided by Mr.Junzo Yana (Institute of Carbon Science and Technology, Shinshu University). All other reagents used for the experiments were commercial or laboratory grade.

\section{Apparatus and electrode}

The CV measurement system was constructed from an electrochemical analyzer (Model 802B, BAS, Tokyo, Japan). All experiments were performed with a conventional threeelectrode system using a modified $\varphi 3.0 \mathrm{~mm}$ Au electrode (BAS) as a working electrode, a platinum wire $(\varphi 1 \mathrm{~mm}, 5 \mathrm{~cm})$ as a counter electrode, and $\mathrm{Ag} / \mathrm{AgCl}$ electrode saturated with $0.5 \mathrm{M} \mathrm{KCl(BAS)}$ as a reference electrode. The measurements were performed in a cell stand (CS-3, BAS) for keep the measurements steadily.

\section{Fabrication of the label-free immunosensor}

The surface of the Au electrode (geometric surface area $=7.1 \mathrm{~mm}^{2}$ ) was polished using diamond paste with $\varphi 1.0 \mu \mathrm{m}$ particles and alumina slurries with $\varphi 0.05-\mu \mathrm{m}$ particles. Polished electrode further cleaned by ultra-sonication in distilled water and dehydrated ethanol sequentially each of $5 \mathrm{~min}$. Cycling the electrode potential in a weak sulfuric acid solution until a stable CV scan is achieved is a very common electrochemical cleaning technique. Working electrode was cycled from 0 to $1500 \mathrm{mV}$ (vs. $\mathrm{Ag} / \mathrm{AgCl}$ ) at a rate of $0.1 \mathrm{~V} / \mathrm{s}$ in $0.5 \mathrm{M}$ sulfuric 
acid for 50 cycled scans until the CV becomes stable. The electrode was then dried in flowing pure nitrogen gas.

\section{Electrode which surface modified with SWCNT}

Fig. 1(a-d) shows the modification steps of the label-free immunosensor which its surface not modified with SWCNT. Treated working electrode was immersed in MPA solution in the dark at room temperature for $8 \mathrm{~h}$. In this step, a SAM formed on the surface of the Au electrode (SAM/Au electrode; Fig. 1a). The electrode was then placed in EDC-NHS solution (EDC $100 \mathrm{mg}^{-1}$, NHS $100 \mathrm{mg}^{-1}$ ) at room temperature for $2 \mathrm{~h}$ to modify a carboxyl group to form an amine-reactive ester (Fig. 1b). The electrode was then rinsed with distilled water to remove non-specific, physically absorbed EDC and NHS and dried under pure flowing nitrogen gas. $0.5 \mathrm{mg} \mathrm{ml}^{-1}$ Polyclonal anti-DHP immunoglobulin $\mathrm{G}(\mathrm{lgG})$ in $0.1 \mathrm{M}$ phosphate buffer $(\mathrm{pH} 7.4)$ was then placed on the modified surface of the working electrode and overnight at $4{ }^{\circ} \mathrm{C}$, thereby forming the $\mathrm{IgG} / \mathrm{SAM} /$ Auelectrode(Fig. 1c). Then, $1.0 \mathrm{mg} \mathrm{ml}^{-1} \mathrm{BSA}$ was applied as a blocking agent to prevent non-specific binding (Fig. 1d). Abbreviated"Sensor A" in the after.

For both of the sensors, After the modification the working electrode was immersed in $15 \mathrm{ml}$ of $5 \mathrm{mMK}_{3}\left[\mathrm{Fe}(\mathrm{CN})_{6}\right]$ solutioncontaining $0.1 \mathrm{M} \mathrm{KCl}$ with a reference electrode and a counter electrode. The electric potential was swept from -0.2 to $+0.6 \vee$ for 20 scans for decide the initial value of anodic peak. At last, the electrode surface dried, it was immersed in $1.0 \mathrm{ml}$ of DHP standard solutions with different concentration in order to make specified immunoreaction for $10 \mathrm{~min}$.

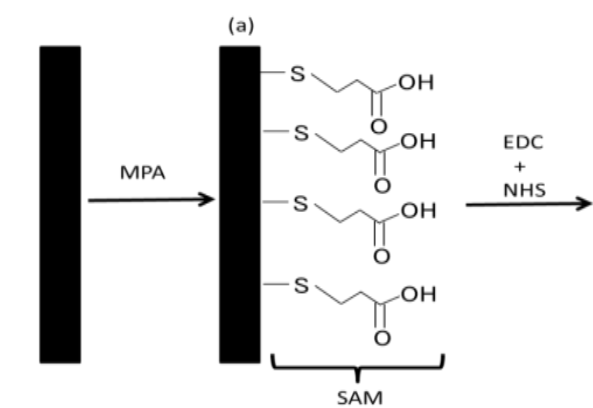

Bare Au

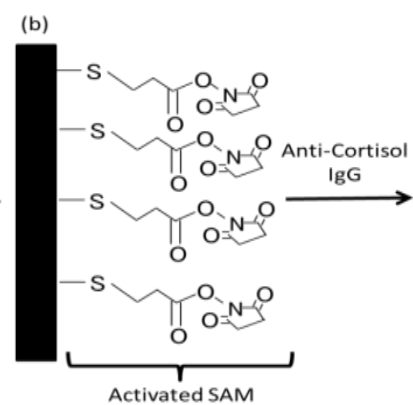

Activated SAM / Au

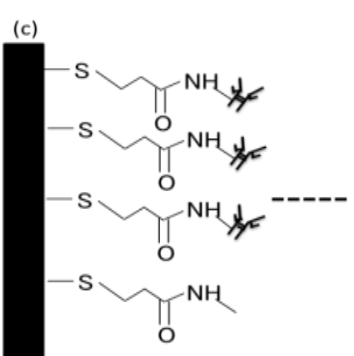

Antibody / SAM / Au

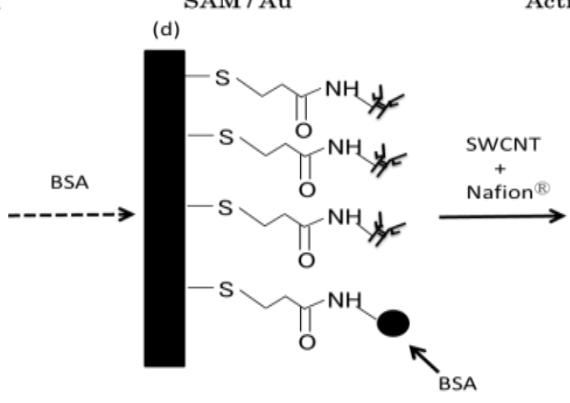

BSA/Antibody / SAM / Au

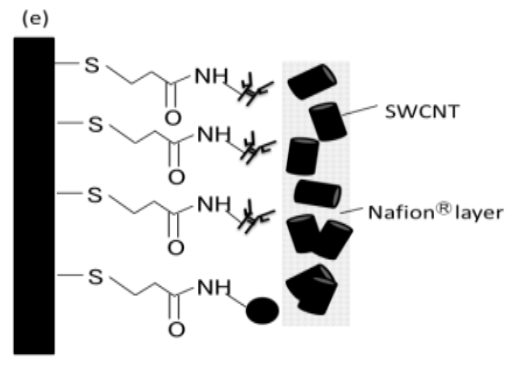

SWCNT / BSA / Antibody / SAM / Au

Figure.1 Schematic diagram of surface modification of the proposed immunosensor: (a) formation of the SAM. (b) Activation of SAM (c) Antibody immobilization (d) Blocking by BSA (Sensor A) (e) Form of SWCNT layer (Sensor B) 


\section{Measurements}

The electrochemical characteristics of the immunosensors were measured by CV. After immunoreaction, the working electrodewas immersed in the same $\mathrm{K}_{3}\left[\mathrm{Fe}(\mathrm{CN})_{6}\right]$ solution and parameters of $\mathrm{CV}$, and the change of the anodic peak current was evaluated.

\section{Specificity of the proposed immunosensor}

The specificity of "Sensor B" was investigated using other steroid hormones which have similar structures to DHP, such as cortisol, estriol, and testosterone. The immunosensor was incubated in $1.0 \mathrm{ml}$ of each hormone solution in the range from 2.0 to $50000.0 \mathrm{pg} \mathrm{ml}^{-1}$ for 10 min. After that, CV was also performed in the same parameters and solution for 20 cycles.

\section{Analysis of goldfish blood plasma}

"Sensor B"was applied to measure DHP levels in goldfish plasma. The goldfish were cultured at the Tokyo University of Marine Science and Technology and cultured in a $500 \mathrm{~L}$ oxygenated tank with a controlled temperature of $22{ }^{\circ} \mathrm{C}$ and kept under a natural photoperiod. First, pituitary extract of Hypophthalmichthysmolitrix, which induces ovulation, was administered at the bottom of the goldfish anal fin, and blood samples were collected every $3 \mathrm{~h}$. The blood samples were immediately centrifuged $(450 \times \mathrm{g}, 10 \mathrm{~min})$ to separate the plasma. Plasma samples $(100 \mu \mathrm{l})$ were transferred to clean test tubes and diluted with $900 \mu \mathrm{l}$ phosphate-buffered saline (PBS, pH 6.5). The diluted plasma sample was diluted 1/50 and frozen at $-20^{\circ} \mathrm{C}$ until use. The measurement of diluted plasma samples was performed in $15 \mathrm{ml}$ of $5 \mathrm{mMK}_{3}\left[\mathrm{Fe}(\mathrm{CN})_{6}\right]$ solution with same parameters mentioned above.

\section{Analysis using ELISA as a conventional method}

DHP levels of the same samples were also determined using ELISA as a conventional method. We use a 96-Well strip Plate (Cayman Chemical) pre-coated with mouse anti-rabbit lgG and prepared blank (Blk), non-specific binding (NSB), maximum binding (Bo), and sample wells. Absorbance of each well at $405 \mathrm{~nm}$ was measured using a Multiskan JX (Thermo Scientific, MA). DHP levels of blood plasma were calculated based on the calibration curve of absorbance, derived by the following equation.

$$
\text { Abs. }(\%)=\frac{\text { ave. Sample }- \text { ave. } B l k-\text { ave. } N S B}{\text { ave. } B_{0}-\text { ave. } N S B}
$$

ave.Sample: average of sample absorbance; ave.Blk: average of blank absorbance ave.NSB: average of non-specific absorbance; ave. $B_{0}$ : average of maximum binding absorbance 


\section{RESULTS AND DISCUSSION}

\section{Electrochemical characteristics of the modified electrode}

In the fabrication of the label-free immunosensor with SWCNT, the electrode was physicochemically modified in several steps. Due to their electrochemical properties, each step shows different current value on the CV diagram. The CV results using each of the modified electrodes are shown in Fig. 2. For "Sensor A", the anodic peak current of the electrode modified with SAM, IgG and BSA (b) was decreased compared with that of the bare electrode (a). On the other hand, with the form of SWCNT layer, the anodic peak current of "Sensor B" increased greatly (c).

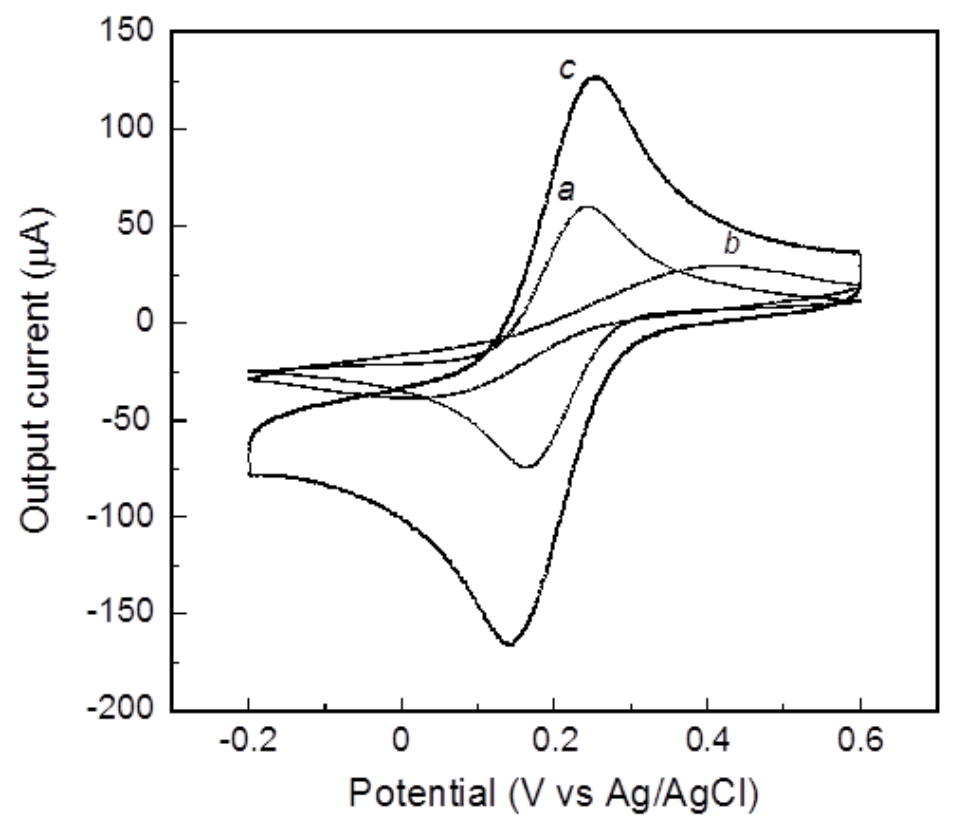

Figure. 2 Electrochemical behavior of the modified electrode (a) Bare Au electrode (b) $\mathrm{BSA} / \mathrm{IgG} / \mathrm{SAM} / \mathrm{Au}$ electrode (c) SWCNT/BSA/IgG/SAM/Au electrode Cyclic voltammograms of differently modified electrodes in $5 \mathrm{mM} \mathrm{K} 3\left[\mathrm{Fe}(\mathrm{CN})_{6}\right]$ with $0.1 \mathrm{M}$ $\mathrm{KCl}$ at a scan rate of $0.1 \mathrm{~V} \mathrm{~S}^{-1}$. All potential are given versus those of an $\mathrm{Ag} / \mathrm{AgCl}$ electrode. The electric potential range was swept from -0.2 to $+0.6 \mathrm{~V}$

To confirm the formation of each molecular layer, we compared the cyclicvoltammograms for each modification on the electrode surface. As shown in Fig. 2, the anodic peak current of the electrode modified with $S A M, \lg G$, and $B S A$ decreased sharply compared with that of bare Au electrode. It indicate that a SAM, IgG and BSA hold a low conductivity then inhibited electron transfer between the electrode surface and oxidation-reduction substances. Accordingly, the results of Fig.2 also suggested that each molecular layer was densely formed on the electrode surface. On the other hands, the anodic peak current increased greatly from 30.1 to $127 \mu \mathrm{A}$ after the immobilization of the SWCNT. It is suggested that the inhabitation of the electron transfer was relieved and the transfer become amplified. Moreover, the anodic peak current of the CV signal before the antigen-antibody interaction was defined as the 
blank, and the deviation between the anodic peak current and the blank was used as the response (output current).

\section{Calibration curve of the proposed immunosensor system}

DHP standard solutions were measured using the proposed sensor system, and the CV of each DHP standard solution is shown in Fig. 3. The anodic peak current decreased with an increase in DHP level. The calibration curve was drawn based on the CV data, in which the $y$ axis shows the current decrease on anodic peak current, and the $x$-axis shows the logarithmic level of DHP (Fig. 4). A good linear correlation curve was obtained between the output current and DHP levels from 7.8 to $500.0 \mathrm{pg} \mathrm{ml}^{-1}$ for the "sensor A" (correlation coefficient: 0.997, Fig. 4a) and 15.6 to $50000.0 \mathrm{pg} \mathrm{ml}^{-1}$ for the "Sensor B" (correlation coefficient: 0.983, Fig. 4b).

Based on the results, DHP levels correlated with the sensor output of "Sensor B" in the range of $15.6-50000.0 \mathrm{pg} \mathrm{ml}^{-1}$ (Fig. 4b). We measured the sensitivity of the proposed Sensor B by calculating the detection limit using a signal/noise ratio of 3 . According to Currie (1968), output current was defined as a signal(S), and the limit of detection (LOD) was defined as three times the standard deviation of the signal of the blank. Thus, the limit of detection was

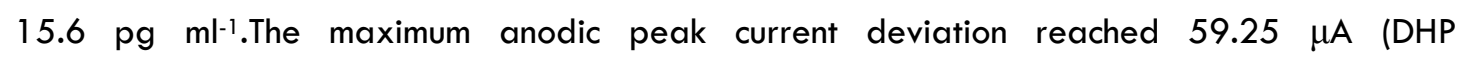
concentration:50000.0 pg ml-1, Fig. 3b).On the other hand, the label-free immunosensor without forming a SWCNT layer, the maximum value of anodic peak current deviation was $7.40 \mu \mathrm{A}$ (DHP concentration: 500 pg ml-1, Fig. 3a).
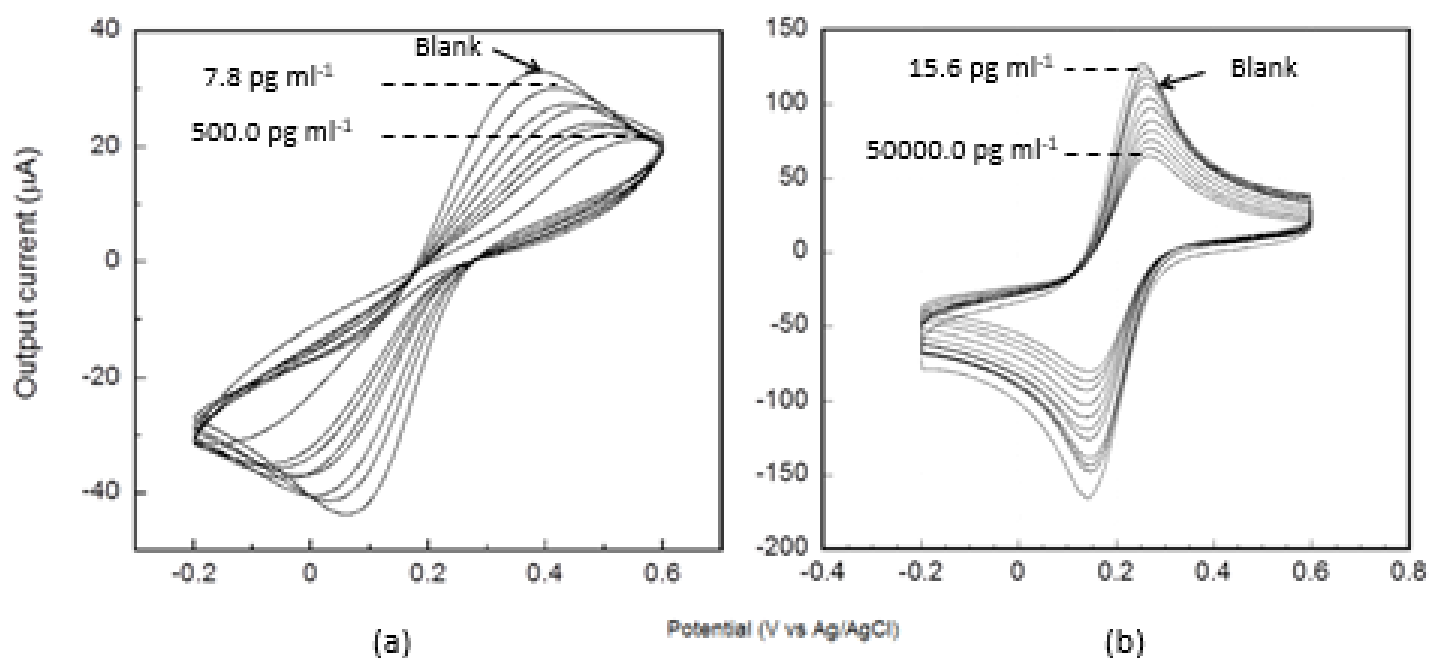

(a)

Figurw. 3 Cyclic voltammograms of differently DHP levels in $5 \mathrm{mMK}_{3}\left[\mathrm{Fe}(\mathrm{CN})_{6}\right]$ with $0.1 \mathrm{M} \mathrm{KCl}$ at a scan rate of $0.1 \vee \mathrm{S}^{-1}$. The electric potential range was swept from -0.2 to +0.6 V.(a)Current Response of "Sensor A" depends on different concentration of DHP. (b)Current Response of "Sensor B" depends on different concentration of DHP. 
DHP levels could only be measured in the range of 7.8-500 pg ml-1. The "Sensor B" improved the dynamic range 100-fold compared to using "Sensor A". Therefore, by forming a layer of SWCNT, the dynamic range of the sensor can be dramatically increased by its high conductivity. Moreover, for both of the sensors, due to additional procedures such as platewashing and adding reagents those are required for the conventional methods are not necessary with this method, one measurement could be completed within $15 \mathrm{~min}$.
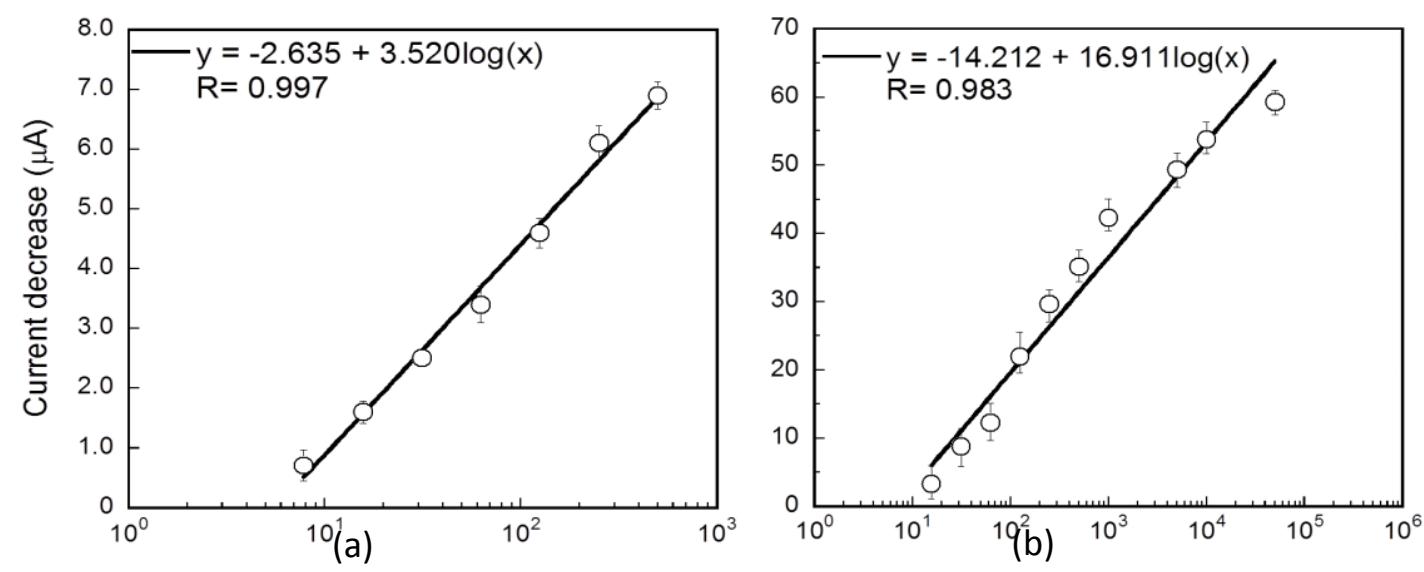

DHP concentration $\left(\mathrm{pg} \mathrm{ml}^{-1}\right)$

Figure. 4 Calibration curve of label-free immunosensor based on Figure. 3 (a) Data based on Figure. 3a. (b)Data based on Fig. 3b.

\section{Specificity of the proposed sensor system}

The specificity of "Sensor B" was investigated using other kinds of steroid hormones which has a similarly structure, such as cortisol, estriol, and testosterone (Figure. 5). The output current decreases gradually increased when measuring a DHP standard solution. On the other hand, the output current decreases of other hormones were kept steady.

As we all known, in fish plasma, there also contains other steroid hormones which has a similarly structure with DHP. Cortisol is a glucocorticoid secreted from interrenal gland and is involved in fish stress response. Estriol is an estrogen secreted from granulosa cells of the ovarian follicles and is involved in the specialization or development of the genital gland (Tamura et al. 1991). Testosterone is an androgen involved in forming spermatozoon, the development of secondary sex characteristics, and the expression of sexual behavior. Moreover, testosterone is a precursor for estrogen synthesis from cholesterol (Kobayashi and Adachi 2002). Therefore, the specificity of the sensor has been investigated. Compared with the measurement data, the other hormones examined did not show a great change in the anodic peak current. It indicated that the proposed label-free immunosensor is sufficiently specific for DHP. 


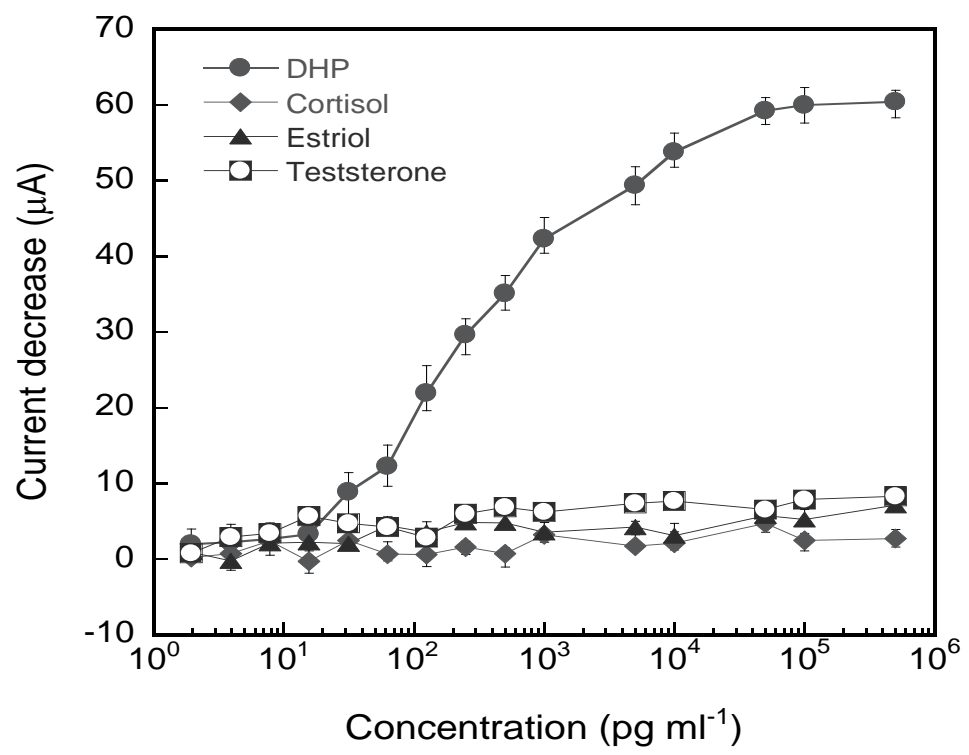

Figure. 5 Relationship between the decrease in the anodic peak current and evaluated steroid hormone concentration. Cyclic voltammograms in $5 \mathrm{mMK}_{3}\left[\mathrm{Fe}(\mathrm{CN})_{6}\right]$ with $0.1 \mathrm{M} \mathrm{KCl}$ at a scan rate of $0.1 \mathrm{~V} \mathrm{~S}^{-1}$. The electric potential range was swept from -0.2 to $+0.6 \mathrm{~V}$. The measurements of each sample were practiced 3 times. The error bar was plotted by using average and maximum (minimum) measurement value

\section{Analysis of goldfish blood plasma}

"Sensor B" was applied to monitor DHP levels in goldfish plasma. Fig. 6 shows the relationship between DHP levels in goldfish plasma measured by the proposed sensor system and by ELISA. A good linear correlation was obtained between the values determined by the proposed sensor system and same samples determined by the conventional ELISA method (correlation coefficient: 0.934)

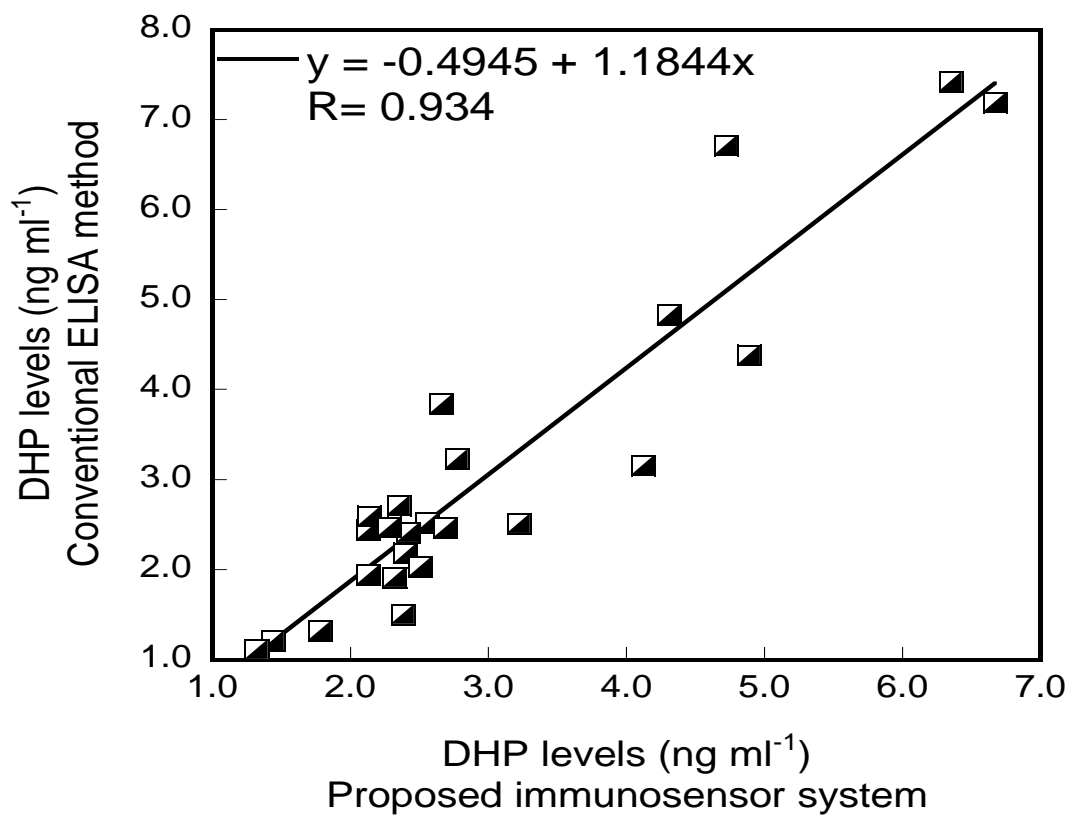

Figure 6 Correlation curve of "Sensor B" with that of conventional ELISA method $(n=25)$. 
From the results, DHP values determined using the proposed label-free immunosensor system strongly correlated with those obtained by ELISA in the range of 0.1-7.7 $\mathrm{ng} \mathrm{m}^{-1}$. Thus, it suggest that the proposed sensor system could be satisfactorily applied to actual fish plasma samples for monitoring DHP levels.

\section{CONCLUSION}

According to described results, the present findings confirm that DHP levels in fish plasma could be measured in a rapid and sensitive way by using the proposed label-free immunosensor. Different from conventional methods, such as ELISA and labeled immunosensor which require long incubation times and some complicated steps, our sensor only take around $15 \mathrm{~min}$ for one sample, including the immunoreaction and CV measurement time. We also believed that we can use the same mechanism to detect other kinds of steroid hormones, such as cortisol, testosterone.

\section{Acknowledgments}

This research was supported in part by a Grant-in-Aid for Scientific Research (B) from The Ministry of Education, Culture, Sports, Science, and Technology. We wish to thank Mr. Junzo Yana (Institute of Carbon Science and Technology, Shinshu University) for their helpful discussions.

\section{REFERENCES}

Asahina K,Kambegawa A, Higashi T. 1995. Development of a micro titer plate enzyme-linked immunosorbent assay for 17, 20 $\beta$ - dihydroxy-4-pregnen-3-one, a teleostgonadal steroid. Fish Sci. 61 : $491-494$

Bromage N, Porter M, Randall C. 2001. The environmental reguluation of maturation in farmed finfish with special referece to the role of photoperiod and melatonin. Aquaculture 197: 63-68

Bulukin E, Mecucci V, Minunni M, Pretti C, Soldani G, Masdini M. 2007. An optical immunosensor for rapid vitellogenin detection in plasma from carp (Cyprinuscarpio).Talanta 72: 785-790

Carmona-Osalde C, Rodriguez-Serna M, Olvera-Novoa MA, Gutierrez-Yurrita PJ. 2004. Gonadal development, growth and survival of the crayfish Procambarusllamasi at three different water temperature. Aquaculture 232: 305-316

Currie LA. 1968. Limits for qualitative detection and quantitative determination: application to radiochemistry. Anal Chem. 40: 586-593

DuboisE.A,SlobS, Zandbergen M.A, Peute J, GoosH.J.Th. 2001. Gonadal steroids and the maturation of the species-specific gonadotropin-releasing hormone system in brain and pituitary of the male African catfish (Clariasgariepinus). Comp. Biochem. Physiol. B 129B: $381-387$

Endo H, Igarashi M, Banba A, Ohnuki H, Ushio H, Hayashi T, Ren H, Yoshizaki G. 2011. Electrode-based immunologic assay system to monitor oocyte maturation-inducing hormone in fish. Int. J. Environ. Anal. Chem. 91 : 174-184

Haussmann MF, Vleck CM, Farrar ES. 2007. A laboratory exercise to illustrate increased salivary cortisol in response to three stressful conditions using competitive ELISA. Adv. Physiol. Educ. 31: 110-115 
Huang CH, Sedlak DL. 1998. Analysis of estrogenic hormones in municipal wastewater effluent and surface water using enzyme-linked immunosorbent assay and gas chromatography/tandem mass spectrometry. Environ. Toxicol. Chem. 20: 133-139

Idler DR, Fagerlund UHM, Ronald AP. 1960. Isolation of pregn-4-ene-17-alpha, 20beta-diol3-one from the plasma of Pacific salmon (Oncorhynchusnerka).Biochem.Biophys. Res. Commun. 2: 133-137

lijima S. 1991. Helical microtubules of graphitic carbon. Nature 354: 56-58

Jarboe HH, Romaire RP. 1995. Effects of density reduction and supplemental feeding on stunted crayfish Procambarusclarkia populations in earthen ponds. J. World Aquac. Soc. 26: $29-37$

Kagawa $H$, Young G, Nagahama Y. 1983. Changes in plasma steroid hormone levels during gonadal maturation in female goldfish Carassiusauratus. Bull. Jpn. Soc. Sci. Fish 49:1783-1787

King VW, Berlimsky LD, Sullivan CV. 1995. Involvement of gonadal steroids in final oocyte maturation of white perch (Moroneamericana) and white bass (M. chrysops): in vivo and in vitro studies. Fish Physiol. Biochem. 14:489-500

Li ZL, Wang S, Lee NA. 2004. Development of a solid-phase extraction-enzyme-linked immunosorbent assay method for the determination of estrone in water. Anal.Chim.Acta. 503:171-177

Lous SW, Aida K, Hanyu I, Sakai K, Nomura M, Tanaka M, Tazaki S. 1984. Endocrine profiles in the females of a twice-annually spawning strain of rainbow trout. Aquaculture 43: 13-22

Mason DW, Williams AF. 1980. The kinetics of antibody binding to membrane antigens in solution and at the cell surface. Biochem. J. 187: 1-20

Matsuyama M. 1997. Maturation inducing hormone of fish. Radioisotopes 46: 263-264

Moriwaki T, Kobayashi M, Aida K, Hanyu I. 1991.Changes in plasma gonadotropin and steroid hormone levels during ovulation induced by HCG treatment in female goldfish. Bull. Jpn. Soc. Sci. Fish 57: 41-43

Nagahama Y, Yamashita M. 1989.Mechanisms of synthesis and action of 17, 20ß-dihydroxy4-pregnen-3-one, a teleost maturation-inducing substance. Fish Physiol. Biochem. 7: 193200

Ohata K, Yamaguchi S, Yamaguchi A, Matsuyama M. 2002.Biosynthesis of steroids in ovarian follicles of red seabream, Pagrus major (Sparidae, Teleostei) during final oocyte maturation and the relative effectiveness of steroid metabolites for germinal vesicle breakdown in vitro.Comp. Biochem. Physiol. B 133B: 45-54

Qiu LP, Wang CC, Hu P, Wu ZS, Shen GL, Yu RQ. 2010. A label-free electrochemical immunoassay for $\lg G$ detection based on the electron transfer. Talanta 83: 42-47

Rivas GA, Rubianes MD, Rodriguez MC, Ferreyra NF, Luque GL, pedano ML, Miscoria SA, Parrado C. 2007. Caron nanotubes for electrochemical biosensing.Talanta 74: 291-307

Saito R, Shinohara H. 2004. Foundation and application of carbon nanotubes.Baifukan Co., Ltd., Tokyo (in Japanese)

Su X, Chew FT, Li SFY. 2000. Piezoelectric quartz crystal based label-free analysis for allergy disease. Biosens.Bioelectron. 15: 629-639

Suzuki R, Yamaguchi M. 1977. Effect of temperature on maturation of a cyprinid loach. Bull. Jpn. Soc. Sci. Fish 43: 367-373

Tamura T, Itazawa Y, Oguri M, Hanyu I. 1991. Fish physiology Introduction. Kouseisha Kouseikaku Co., Ltd., Tokyo

Truscott B, Idler DR, So YP, Walsh JM. 1986. Maturational steroids and gonadotropin in upstream migratory sockeye salmon. Gen. Comp. Endocrinol. 62: 99-1 10 
Ulman A. 1998. Thin films: Self-assembled monolayers of thiols (thin films). Academic Press, New York, pp 45-65

Vidal JC, Espuelas J, Garcia-Ruiz E, Castillo JR. 2004. Amperometric cholesterol biosensors based on the electropolymerization of pyrrole and the electrocatalytic effect of Prussian-Blue layers helped with self-assembled monolayers. Talanta 64: 655-664

Viswanathan S, Wu L, Huang M, Ho JA. 2006. Electrochemical immunosensor for cholera toxin using liposomes and poly (3,4-ethylenedioxythiophene)-coated carbon nanotubes. Anal. Chem. 78: $1115-1121$

Yamauchi K, Kagawa H, Ban M, Kasahara Y. 1984. Changes in plasma estradiol-17 $\beta$ and 17, $20 \beta$ - dihydroxy-4-pregnen-3-one levels during final oocyte maturation of the masu salmon, Oncorhynchusmasou. Nippon Suisan Gakkaishi 50: 2137

Zhang L, Yuan R, Huang X, Chai Y, Tang D, Cao S. 2005. A new label-free amperometricimmunosensor for rubella vaccine.Anal.Bioanal. Chem. 381: 1036-1040 